\title{
A Pictorial Disease Severity Key and the Relationship Between Severity and Incidence for Black Root Rot of Pansy Caused by Thielaviopsis basicola
}

\author{
Warren E. Copes, USDA-ARS Southern Horticultural Laboratory, Poplarville, MS 39470; and Katherine L. \\ Stevenson, Department of Plant Pathology, University of Georgia, Tifton, GA 31793
}

\begin{abstract}
Copes, W. E., and Stevenson, K. L. 2008. A pictorial disease severity key and the relationship between severity and incidence for black root rot of pansy caused by Thielaviopsis basicola. Plant Dis. 92:1394-1399.

A pictorial key was developed and the relationship between disease severity $(S)$ and incidence $(I)$ was examined to aid in the assessment of black root rot of pansy caused by Thielaviopsis basicola. The key consisted of photographs of root segments that represented nine disease severity levels ranging from 1 to $91 \%$. Pansies that had received different fertility treatments, as part of seven separate experiments, were inoculated with T. basicola. Four weeks after inoculation, roots were washed, and incidence and severity of black root rot were visually assessed using a grid-line-intersect method. Disease incidence ranged from 1.3 to $100 \%$, and severity ranged from 0.1 to $21.4 \%$ per plant. Four different mathematical models were compared to quantitatively describe the $I-S$ relationship for the combined data from all seven experiments. Although all models provided an adequate fit, the model that is analogous to the Kono-Sugino equation provided the most reliable estimate of severity over the entire range of disease incidence values. The predictive ability and accuracy of this model across data sets was verified by jackknife and cross-validation techniques. We concluded that incidence of black root rot in pansy can be assessed more objectively and with greater precision than disease severity and can be used to provide reliable estimates of disease severity based on derived regression equations that quantify the $I-S$ relationship for black root rot.
\end{abstract}

Additional keywords: Chalara basicola, disease intensity, disease measurement, rating scale

Black root rot of pansy (Viola $\times$ wittrockiana Gams ex Kappert) is caused by Thielaviopsis basicola (Berk. \& Broome) Ferraris. The synanamorph of $T$. basicola is Chalara elegans Nag Raj \& Kendrick. Black root rot and Thielaviopsis root rot are common names used for disease caused by the same pathogen on multiple ornamental and agricultural crops $(14,16)$. The macroscopic appearance of the disease on infected root sections ranges from a characteristic flat black, for which the disease is named, to degrees of brown necrosis that may include interspersed black areas. The visual appearance of the root disorder results from a combination of the symptom of root necrosis and sign of melanized aleuriospores produced on the surface and internally in infected tissue (2). Black root rot disease typically has been assessed using a measure of severity (the proportional or percent area of plant parts or plants diseased) for an entire root

Corresponding author: W. E. Copes

E-mail: warren.copes@ ars.usda.gov

Accepted for publication 27 June 2008.

doi:10.1094/PDIS-92-10-1394

This article is in the public domain and not copyrightable. It may be freely reprinted with customary crediting of the source. The American Phytopathological Society, 2008. system based on the researcher's preference of a qualitative descriptive scale (1) or quantitative scale of percent disease $(4,12,34)$.

Qualitative descriptive keys, quantitative percentage scales, and standard area diagrams can be helpful for obtaining accurate and repeatable estimates of disease intensity. However, efficiency, reproducibility, and precision of the assessment method will depend on the clarity of the description or scale to the evaluator, the nature and extent of disease symptoms and signs, experience of the evaluator, and the objective of the research $(5,18-20,23,24)$. All of the methods involve some degree of subjectivity. Most assessment methods have been developed for foliar diseases (20). Qualitative descriptive keys and quantitative percentage scales have been used commonly for assessing disease intensity (a generalized quantity of disease) on roots, but few disease rating scales have been developed. The appropriateness of a scale will vary with the host plant and disease. O'Brien and van Bruggen (24) found that a qualitative scale assessed on mature plants was generally the most accurate and precise for assessing severity of corky root of lettuce, but the quantitative Horsfall-Barratt scale was most accurate for roots in the 20 to $80 \%$ severity range.

A measurement of incidence (the proportional or percentage number of plant parts or plants diseased) generally requires less time except at intensity levels close to zero when detection of early stages of signs and symptoms are important. Estimates of incidence are generally more precise than estimates of severity, and could provide an alternative assessment approach that may be transformed into a severity measurement when a mathematical relationship exists between the two. As with the previously mentioned assessment scales, quantitative relationships between incidence and severity have been shown mainly with foliar diseases $(6,9,13,26$, 28,30). Mathematical relationships have been identified between disease intensity and soil inoculum density in cotton fields (12), lesions on crowns and subcrown internodes of wheat (31), lesions on lower stems of canola (32), yield of lettuce (24), and host resistance (3). However, the quantitative relationship between severity and incidence has not been reported previously for a root disease.

Imprecision of foliar disease assessments has been attributed partly to limitations of human visual acuity, including perception of color and optical skewness of the percent light and dark areas between diseased and healthy plant tissue (18-20). Most scales and standard area diagrams have been developed on relatively uniform plant leaves. Disease is more difficult to assess on leaf organs comprised of multiple leaflets, such as bracken fern (19). Although there is no documentation of problems associated specifically with visual perception of root diseases, a number of inherent features of root diseases make assessment a challenging task. Due to the subterranean growth habit, the often difficult step of physically separating roots from the surrounding growing medium is required to obtain an unobstructed view of the entire root system. Even with the aid of more modern image analysis tools, assessment is complicated by the fact that whole root systems are complex threedimensional organs with no significant planar surfaces. If disease severity is evaluated while roots are interspersed within the growing medium, it may be difficult to distinguish the brown discoloration of diseased root tissue from the naturally brown color of roots of some plant species and the brown coloration of rooting media (e.g., pine bark, peat moss). Healthy roots of some annuals, such as pansy, are small in diameter and can have a 
translucent nature that deceptively transmits dark coloration from the rooting media (W. E. Copes, unpublished). Visual assessment of the diseased root system may not provide the quantitative accuracy needed to analyze small treatment differences that could be important in evaluating complex interactions among the pathogen, host, and environment.

The objective of this study was to develop a reliable and efficient method of assessing intensity of black root rot of pansy. Disease incidence and severity were visually assessed using a line-intersect method as a sampling protocol to define root units. A standardized pictorial key is presented as a guide to estimate severity, and the quantitative relationship between incidence and severity was examined.

\section{MATERIALS AND METHODS}

Plant culture. Pansy 'Universal Mixed' (Park Seed Co., Greenwood, SC) seedlings were grown from seed in an autoclaved fine sand and transplanted, at fourth to fifth leaf, into $10-\mathrm{cm}$ pots filled with 250 $\mathrm{ml}$ of an autoclaved fine sand. Sand was autoclaved twice at $125^{\circ} \mathrm{C}$ for $60 \mathrm{~min}$, and cooled to room temperature between heating events. After transplanting, plants were grown in a greenhouse environment at day/night temperatures of $26^{\circ} \mathrm{C} / 22^{\circ} \mathrm{C}$ and alternatively watered or fertilized daily or as needed for 7 weeks. Seven experiments were performed to study the effects of various fertility treatments on disease caused by $T$. basicola, of which three were published (7).

Inoculation procedure. An isolate of $T$. basicola (Tb0024) obtained from diseased pansy roots was grown on V8 agar under continuous light $\left(32 \mu \mathrm{mol} \cdot \mathrm{m}^{-2} \cdot \mathrm{s}^{-1}\right)$ from one 15-W cool-white fluorescent lamp for 14 to 21 days at $20^{\circ} \mathrm{C}$. Endoconidia were obtained by flooding cultures with sterilized deionized water, rubbing the agar surface with a rubber policeman, then filtering through four layers of sterile cheesecloth. Spore concentrations, as determined with a hemacytometer, were adjusted to $5 \times 10^{5}$ endoconidia per liter. Three weeks after transplanting, roots were inoculated with $100 \mathrm{ml}$ of spore suspension, which was poured into the top of the pot and allowed to drain through the sand.

Disease assessments. A pictorial disease assessment key was created using photographs of pansy root segments exhibiting a wide range of disease severities taken at $\times 100$ magnification. The root segment was cut from the photograph, then the areas of melanized root cells and aleuriospores were cut from the photograph. Disease severity was estimated as the weight of the diseased portion of the root segment photograph as a percentage of the total weight of the root segment photograph, with weight measured to the nearest $0.001 \mathrm{~g}$. Photographs depicting 0,1, 4, 8, 17, 33, 56,71 , and $96 \%$ disease severity were selected to include in the pictorial key (Fig. 1).

Four weeks after inoculation, each plant was removed from the pot, and roots were carefully washed free of sand with tap water using a variable pressure nozzle on a hose, placed into a plastic bag, and held at $5^{\circ} \mathrm{C}$ for a maximum of 2 days. All individual roots comprising the entire root system were detached from each plant. A 1-g subsample of detached root lengths was arbitrarily selected from each root system, placed in a plastic perforated specimen container, and immersed in a preservative solution $(950 \mathrm{ml}$ of $95 \%$ ethanol, $90 \mathrm{ml}$ of $85 \%$ lactic acid USP, and $950 \mathrm{ml}$ of deionized water). The preservative had no noticeable effects on root turgor, root necrosis, or spore turgor.

The 1-g subsample of roots was cut into 3-mm lengths immediately prior to making disease intensity assessments. Roots were held beside a plastic ruler and cut into approximately 3-mm lengths with stainless steel surgical scissors. Roots were mixed thoroughly, and evenly distributed in the bottom half of a $100 \times 15 \mathrm{~mm}$ disposable petri dish. This was placed on top of a transparency imprinted with perpendicular grid lines spaced $2.54 \mathrm{~cm}$ apart and set in the top half of a $100 \times 15 \mathrm{~mm}$ glass petri dish. Disease severity was assessed through a stereoscope at $\times 40$ magnification on an approximately $600-\mu \mathrm{m}$ length of root segment centered at the point it intersected a grid line (10). Root width averaged 230 $\mu \mathrm{m}$ wide and ranged from 150 to $400 \mu \mathrm{m}$. A minimum of 25 root segments was assessed on each of four grid lines for a total of at least 100 root segments per subsample (10). Sample sizes were arbitrarily selected and are the minimum number of values assessed. Disease severity was assessed on a 0 to $100 \%$ scale with intervals of $1 \%$ using the previously described photographic key as a guide. Disease severity $(S)$ of a whole root system was expressed as the sum of disease severity values over all root segments, divided by the total number of root segments assessed per root system. Disease incidence $(I)$ was calculated as the number of root segments with a severity greater than zero divided by the total number of root segments assessed per root system.

Statistical analysis. Four different empirical models were evaluated to describe the quantitative relationship between $I$ and $S$ of black root rot of pansy. For ease of parameter estimation and comparison of parameter estimates, linearized forms of the models were used. The models selected for evaluation were:

$S=\mathrm{a} I+\mathrm{b} I^{2}+\mathrm{c} I^{3}$

$\ln S=\ln (\mathrm{a})+\mathrm{b} \operatorname{CLL}(I)$

$S=\mathrm{a} \ln (1-I)$

$\ln S=\mathrm{a}+\mathrm{b} I$

where $S=$ severity (proportion), $I=$ incidence (proportion), CLL $(I)=\ln [-\ln (1-I)]$ is the complimentary log-log transformation of $I$, and $\mathrm{a}, \mathrm{b}$, and $\mathrm{c}$ are parameters. Each of the candidate models represents a different family of empirical models: the polynomial family (eq. 1), the Poisson family (eq. 2), the restricted exponential family (eq. 3), and the logarithmic family (eq. 4) (21). All of these models have been used to describe similar relationships between disease incidence and disease severity or density (number of lesions or pustules per area of plant parts or soil) for foliar diseases (21), but none have been previously used to describe the quantitative relationship between disease incidence and severity (or density) for a root disease. The four models were fitted to the combined data set, and parameters were estimated by linear regression using the REG procedure of SAS (SAS version 9.1, SAS Institute Inc., Cary, NC).

Based on evaluations of the statistical goodness of fit to the combined data set, the ability of the model to reliably predict severity over the entire range of disease incidence values, the mathematical properties of the model, and meaningfulness of parameters, equation 2 was selected for further evaluation. The predictive ability and accuracy of this model were tested using the jackknife and cross-validation techniques proposed by Jones and Carberry (15) for the development and validation of simulation models. The linearized regression model (eq. 2) was fitted to combined data from seven independent data sets to obtain overall parameter estimates $(\mathrm{P})$ and associated standard errors $(\mathrm{SE})$. The partial values $\left(\mathrm{P}_{(\mathrm{i})}\right)$ of the estimated parameters, $\ln (\mathrm{a})$ and $\mathrm{b}$, were obtained by fitting the model to each of seven resampled data sets (i.e., when each of the seven data sets was singly omitted at a time). Using formulas given in Jones and Carberry, the mean of the partial values $\left(\mathrm{P}_{(.)}\right)$, the first-order jackknife estimates $\left(\mathrm{P}^{*}\right)$ and standard errors ( $\left.\mathrm{SE}^{*}\right)$, and crossvalidation estimates were calculated. The predictive accuracy of the model was examined by comparing the prediction variances and coefficients of determination from the cross-validation technique and the overall model fitted to the combined data from all seven experiments.

\section{RESULTS}

The pictorial key shows a range of severity values for individual root segments from 0 to $91 \%$ (Fig. 1). While the maximum $S$ value in this study for a whole root system was $21.4 \%$, the range of severity values for individual root segments ranged from 0 to $97 \%$. For a typical root system, approximately $85 \%$ of the root segments with symptoms had a severity value less than $35 \%$, and less than $4 \%$ of them had a severity value greater than $50 \%$ (data not shown).

All of the candidate models provided a relatively good fit to the combined disease 
incidence and severity data and an adequate description of the relationship between incidence and severity of black root rot (Table 1). Predicted and observed severity values as a function of observed disease incidence for the four models are shown in Figure 2. Goodness of fit statistions are shown in Table 1. Of the four model (eq. 1) provided the best statistical fit to the data, based on good agreement between the predicted and observed disease severity values $\left(r^{2}=0.759, \mathrm{MSE}=\right.$ $0.00041)$. However, even with three parameters, the model still consistently overestimated severity at low levels of disease tics and a summary of residual plot evaluacandidate models, the cubic polynomial

incidence $(I<0.20)$ (Fig. 2A), and residual plots showed a significant fan-shaped pattern, suggesting increasing variation in severity with increasing incidence. Equations 2, 3, and 4 also fit the combined data well. Agreement between predicted and observed severity values for these three models was good, and coefficients of determination were only slightly lower than for equation 1. Equation 3 consistently underestimated severity when disease incidence was less than 0.50 (Fig. 2C). Equation 4 tended to overestimate severity when disease incidence was very low $(I<$ $0.15)$ and underestimate severity when disease incidence was between 0.25 and 0.50 (Fig. 2D). Of the models with fewer
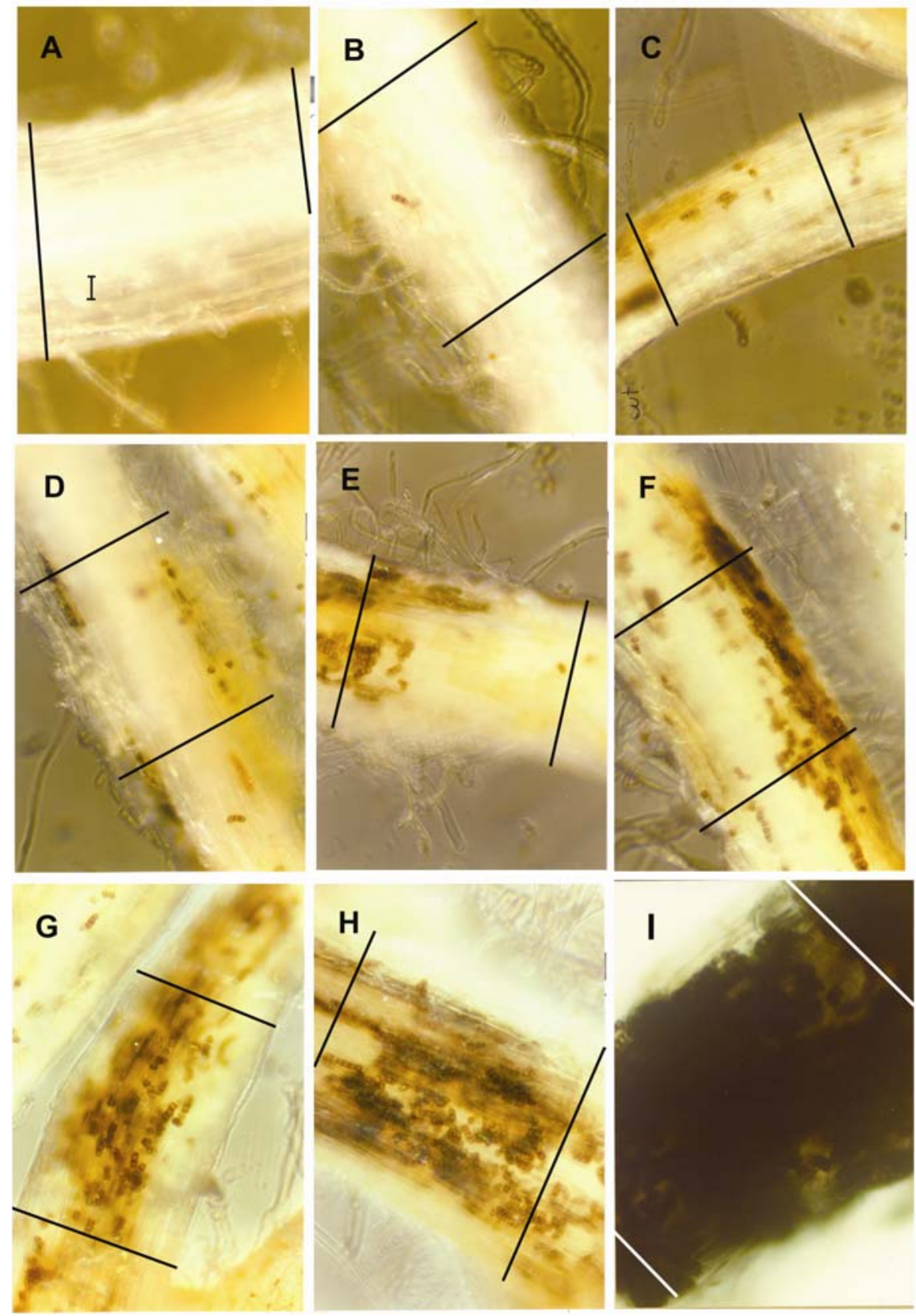

Fig. 1. Root segments of pansy (Viola $\times$ wittrockiana) inoculated with Thielaviopsis basicola exhibiting black root rot severity levels of A, $0 \%$ (not infected), B, $1 \%, \mathbf{C}, 4 \%, \mathbf{D}, 8 \%, \mathbf{E}, 17 \%, \mathbf{F}, 33 \%, \mathbf{G}$, $56 \%$, H, $71 \%$, and I, $91 \%$, based on a combination of signs (aleuriospores) and/or symptoms (necrosis). Severity values were assessed for the area between the lines crossing the root width. Bar $=20 \mu \mathrm{m}$. than three parameters, equation 2 appeared to provide the most consistently reliable estimates of severity over the entire range of disease incidence values in the combined data set (Fig. 2B).

The predictive ability and accuracy of equation 2 to describe the relationship between $I$ and $S$ of black root rot of pansy were verified by jackknifing and crossvalidation techniques. The first-order jackknife estimate of the regression model parameters differed only slightly from the estimates obtained from the combined data set and the resampled data sets (Table 2). The cross-validation prediction variance (0.0006882) was only slightly higher than the overall prediction variance (0.0005924), and the cross-variance coefficient of determination (0.684) was only slightly lower than the overall coefficient of determination (0.779).

\section{DISCUSSION}

The very nature of roots and root diseases presents a challenge for disease assessment. Unlike plant foliage, there is no obvious discrete sampling unit of the root system that would be analogous to a leaf. I is generally simpler and faster to assess than $S$, at least for foliar diseases. If a quantitative relationship between $I$ and $S$ can be established for a given disease, this relationship can be used to estimate $S$ from the more easily measured $I$ values. However, with the exception of two early studies, one on root knot (11) and the other on clubroot of cabbage (25), there is almost no published research on the quantitative relationship between $I$ and $S$ for root diseases. In this paper, we describe a root-line intersect method for assessing intensity of black root rot of pansy, caused by Thielaviopsis basicola, a pictorial key for assessing $S$ levels, and a predictive equation that describes the quantitative relationship between disease $I$ and $S$ that can be used to reliably estimate $S$ from observed $I$ data.

One reason for investigating the $I-S$ relationship is to help determine an assessment method that provides the appropriate precision for the least effort. $I$ is generally simpler and faster to assess than $S$. However, the response pattern often described with the $I-S$ relationship shows selection of $I$ or $S$ as the assessment might depend on whether disease intensity falls below or above a threshold level. Based on the literature, both $I$ and $S$ are often assessed for foliar diseases, whether or not the relationship between $I$ and $S$ is investigated, because both $I$ and $S$ may be statistically relevant for multiple aerial plant units (specified area of leaf, whole leaf, leaves of a specified age, whole plant). The accessibility of units is an important factor that affects selection of assessment approaches with root diseases. For most research on root diseases, $S$ has been assessed using an ordinal or nominal rating scale that is applied to the entire root sys- 
tem, whether separated or not from the growing medium, with no correlation to $I$. Unlike plant foliage, there is no obvious discrete sampling unit of the root system that would be analogous to a leaf. Therefore, sampling units must be explicitly defined and an appropriate sampling protocol devised. The line-intersect method, which was originally used as a simplified method for calculating root length, provides a simple method for sampling $I$ without defining a specific root unit (10). For black root rot of pansy, we propose that I assessed on multiple root units would provide a more precise measure of disease intensity than $S$ assessed either as a single value for an entire root system or on multiple units of a root system. Estimates of $I$ are generally more precise than those of $S$ because $I$ can be assessed objectively. However, assessing $I$ on multiple root segments would take more time than assessing $S$ as a single rating value per root system.

Another reason for investigating the $I-S$ relationship is to understand how various factors influence the $I-S$ relationship over time. Slopes of the $I-S$ relationship of foliar diseases were significantly different in response to cultivar and disease progress $(26,30)$, to climatically distinct locations (6) and seasons (29). These factors were not tested in the present study of T. basicola. Currently, many root diseases are still assessed at a single time. Like many other root diseases, $I$ and $S$ of black root rot of pansy in this study were assessed at only a single point during the epidemic (4 weeks after inoculation). Additional research

Table 1. Fitted regression equations that describe the quantitative relationship between disease severity $(S)$ and incidence $(I)$ of black root rot of pansy, caused by Thielaviopsis basicola, for combined data from seven independent experiments $(N=224)$

\begin{tabular}{llccl}
\hline Equation & Fitted regression equation & $\begin{array}{c}\text { Coefficient of } \\
\text { determination }^{\mathbf{b}}\end{array}$ & $\begin{array}{c}\text { Mean square } \\
\text { error }^{\mathbf{b}}\end{array}$ & Residual plot $^{\mathbf{c}}$ \\
\hline 1 & $S=0.1816 I-0.3843 I^{2}+0.3514 I^{3}$ & 0.759 & 0.00041 & $\begin{array}{c}\text { Fan-shaped pattern, larger magnitude residuals } \\
\text { at } I>50 \% ; \text { mostly negative residuals at } I<20 \%\end{array}$ \\
2 & $\begin{array}{l}\ln S=-2.9397+0.7672 \text { CLL}(I) \\
S=-0.0434 \ln (1-I)\end{array}$ & 0.747 & 0.00046 & $\begin{array}{l}\text { OK; no obvious pattern } \\
\text { Slight fan-shaped pattern; mostly positive residuals } \\
\text { at } I<50 \%\end{array}$ \\
4 & $\ln S=-4.7850+2.8350 I$ & 0.730 & 0.00064 & $\begin{array}{c}\text { Slight sine-wave pattern; large magnitude negative } \\
\text { residuals at } I<10 \%\end{array}$ \\
\hline
\end{tabular}

${ }^{a}$ Disease incidence $(I)$ and severity $(S)$ were expressed as proportions. CLL $(I)=\ln [-\ln (1-I)]$. Model parameters estimated by linear regression (SAS PROC REG).

${ }^{\mathrm{b}}$ Coefficient of determination $\left(r^{2}\right)$ and mean square error (MSE) from linear regression of back-transformed predicted severity values on observed severity values.

${ }^{c}$ Plots of Studentized residuals versus observed disease incidence values.
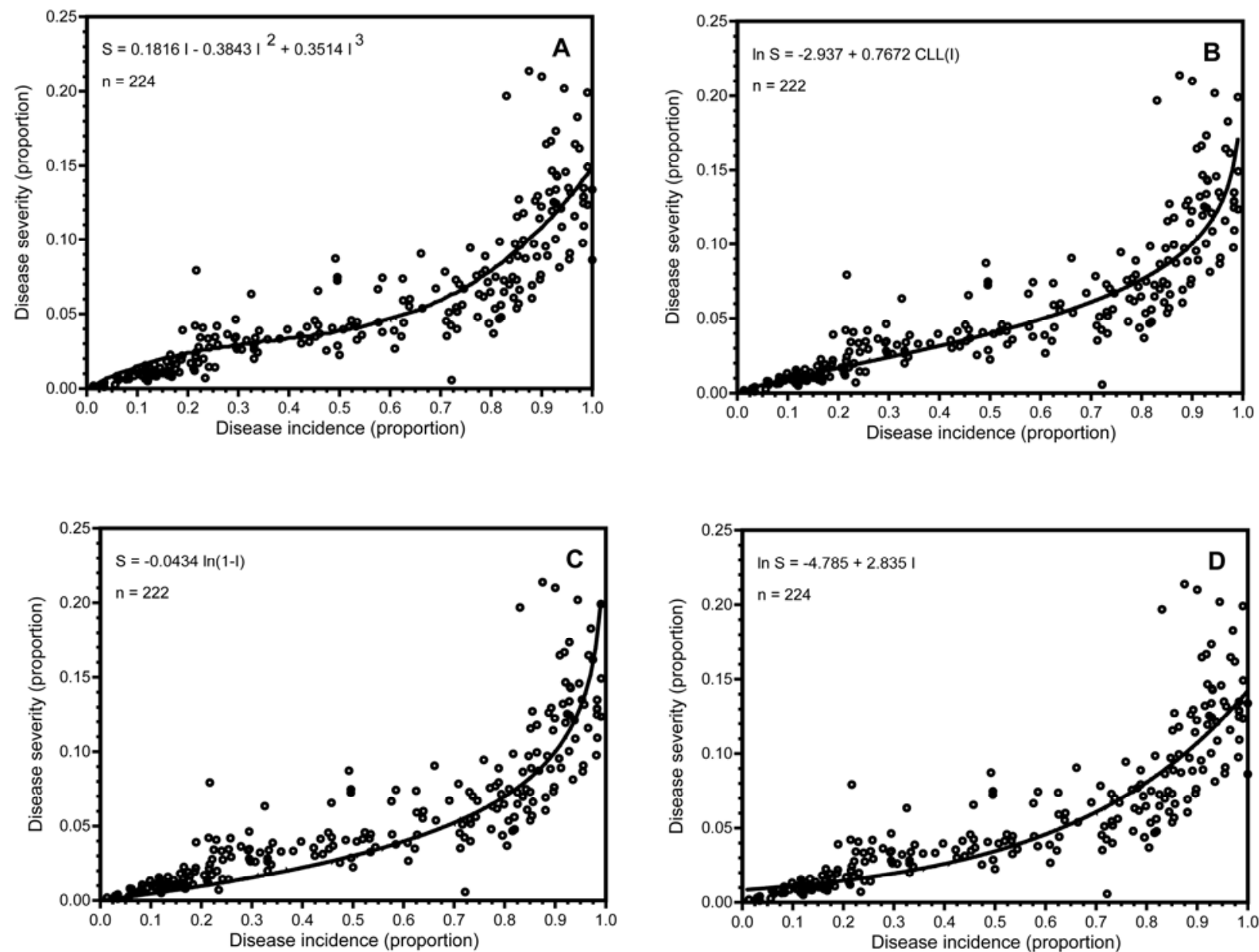

Fig. 2. Comparison of four models to describe the quantitative relationship between severity $(S)$ and incidence $(I)$ of black root rot of pansy $($ Viola $\times$ wittrockiana) inoculated with Thielaviopsis basicola. $\mathbf{A}, S=\mathrm{a} I+\mathrm{b} I^{2}+\mathrm{c} I^{3}$, where a, b, and c are parameters, $\mathbf{B}, \ln S=\ln (\mathrm{a})+\mathrm{b} \operatorname{CLL}(I), w h e r e \operatorname{CLL}(I)=$ $\ln [-\ln (1-I)]$ and a and $\mathrm{b}$ are parameters, $\mathbf{C}, S=\mathrm{a} \ln (1-I)$, where a is a parameter, and $\mathbf{D}, \ln S=\mathrm{a}+\mathrm{b} I$, where a and b are parameters. 
would be required to define factors that may affect the $I$ and $S$ relationship at different spatial scales and degrees of disease aggregation $(28,35)$ and link the rates of change of $I$ and $S$ (33).

Based on past studies of quantitative $I-S$ relationships for foliar diseases, $I$ often increases faster than $S$ during the course of an epidemic. Maximum $I$ and $S$ values, respectively, were 48 and $12 \%$ for banana leaf spot (6), 92 and $12 \%$ for common rust on sweet corn (9), 100 and $8 \%$ for common rust on sweet corn (26), 90 and 3\% for powdery mildew on winter wheat (13), and 90 and $6 \%$ for leaf rust on winter wheat (13). Plotting $I$ as a function of $S$ often yields a typical "saturation curve" pattern (21), in which $I$ increases very rapidly relative to $S$ at low levels of $S$, but eventually reaches a saturation point and levels out as $S$ increases and $I$ approaches $100 \%$. The point at which $I$ levels out and $S$ begins to increase faster relative to $I$ has been used as a threshold level to initiate a control action. The threshold level to signal a first fungicide application of the season was $I=80 \%$ to control common rust powdery mildew on winter wheat (13).

In this study, we investigated the quantitative relationship between $I$ and $S$ of black tions representing four different families of mathematical models. Based on a statistical evaluation and comparison of the four candidate models, a cubic polynomial equation (eq. 1) provided the best predictive relationship between $I$ and $S$ of black root rot of pansy. Similar polynomial relationships between $I$ and $S$ have been demonstrated for several foliar diseases $(6,9,13,26,28,30)$. With three parameters, this was the most complex of the models we considered. Furthermore, the relationship is purely empirical and the three parameters have virtually no biological interpretation, which limits our ability to make any generalizations about the biological mechanisms underlying the $I-S$ relationof sweet corn (9) and $I=65 \%$ to control root rot by fitting relatively simple equa-

ship. However, because this model provided the best statistical fit to our combined data, it may be most useful for estimating disease severity from observed incidence for the purposes of disease assessment.

The other three models evaluated provided similarly good fits to the $I-S$ relationship. Equation 4, representing the logarithmic family of equations, has been used to describe the relationship between the number of uredinia per leaf (disease density) and the percentage of diseased leaves (disease incidence) for common maize rust on sweet corn (9). This model provided an adequate description of the quantitative relationship between $I$ and $S$ for black root rot of pansy and therefore could be useful for disease assessment purposes. However, like the polynomial model (eq. 1), it is also essentially a purely empirical model, and provides little insight into the underlying biology of the $I-S$ relationship. The models representing the Poisson family (eq. 2) and the restricted exponential family (eq. 3) provided equally good statistical fits to the combined $I-S$ data for black root rot of pansy. Equation 3, and its inverse, have been used to describe the relationship between $I$ and $S$ for powdery mildew $(13,27)$ and leaf rust of wheat (13).

Equation 2 is analogous to the KonoSugino equation:

$\ln m=\ln (\mathrm{a})+\mathrm{b} \operatorname{CLL}(I)$

which has been widely employed in entomology to estimate the mean pest population density $(\mathrm{m})$ from the incidence of infestation $(I)$, but has received relatively little attention from plant pathologists (17). The model (or its inverse) has been used to describe the relationship between disease density and disease incidence for wheat powdery mildew (8), apple powdery mildew (36), and Eastern filbert blight (21); and to describe the relationship between $I$ and $S$ for wheat powdery mildew and spear rot of broccoli (21). Our use of equation 2 to model the relationship between $I$ and $S$

Table 2. Parameter estimates and standard errors of a regression model describing the quantitative relationship between disease incidence and severity of black root rot of pansy, caused by Thielaviopsis basicola, using the combined data set from seven independent experiments and resampled data sets from six experiments ${ }^{\mathrm{a}}$

\begin{tabular}{llcc}
\hline & & $\ln (\mathbf{a})$ & b \\
\hline $\mathrm{P}_{(1)}$ & Partial estimate, experiment 1 omitted & -2.9308 & 0.7556 \\
$\mathrm{P}_{(2)}$ & Partial estimate, experiment 2 omitted & -2.9305 & 0.7879 \\
$\mathrm{P}_{(3)}$ & Partial estimate, experiment 3 omitted & -2.8621 & 0.7998 \\
$\mathrm{P}_{(4)}$ & Partial estimate, experiment 4 omitted & -2.9723 & 0.7420 \\
$\mathrm{P}_{(5)}$ & Partial estimate, experiment 5 omitted & -2.9608 & 0.7759 \\
$\mathrm{P}_{(6)}$ & Partial estimate, experiment 6 omitted & -2.9594 & 0.7556 \\
$\mathrm{P}_{(7)}$ & Partial estimate, experiment 7 omitted & -2.9540 & 0.7561 \\
$\mathrm{P}_{\mathrm{SE}}$ & Overall estimate, combined data & -2.9397 & 0.7672 \\
$\mathrm{P}_{(.)}$ & Overall standard error & 0.0289 & 0.0222 \\
$\mathrm{P}^{*}$ & Mean of partial estimates & -2.9386 & 0.7676 \\
$\mathrm{SE}$ & First-order jackknife estimate & -2.9464 & 0.7653 \\
\hline
\end{tabular}

${ }^{a}$ Model (eq. 2 in Table 1): $\ln S=\ln ($ a) + b CLL $(I)$, where disease severity $(S)$ and incidence $(I)$ are expressed as proportions, $\operatorname{CLL}(I)=\ln [-\ln (1-I)]$, and a and $\mathrm{b}$ are parameters. $\ln (\mathrm{a})$ and $\mathrm{b}$ were estimated by linear regression. for black root rot of pansy is the first report of the application of this model to describe a root disease.

The inverse of equation 5 corresponds to the relationship:

$I=1-e^{\alpha m^{\beta}}$

where $\alpha$ and $\beta$ are parameters. In the special case when $\alpha=1$ and $\beta=1$, equation 6 reduces to the discrete Poisson probability distribution function, which describes random counts $(21,22,36)$. Because of this mathematical relationship to the Poisson distribution, equation 2 may therefore seem inherently inappropriate for modeling $S$, which is generally expressed as a continuous random variable, such as proportion or percent diseased area. However, if disease density is expressed as the number of lesions per unit area of leaf, then $S$ is the product of disease density and the mean individual lesion area (21). The model is still essentially empirical, but is appealing from a mathematical perspective because of these underlying distributional properties that provide a link between disease density (i.e., lesion counts), $S$ and I. It is interesting to note that in all cases cited above, where equation 2 has been used to model the relationship between incidence and density or $I$ and $S$, the estimated $\mathrm{b}$ parameter value was very close to $1(0.838<\mathrm{b}<1.14)$. In contrast, when used to describe the relationship between $I$ and $S$ for black root rot of pansy, the overall and jackknife estimates of $b$ were 0.7672 and 0.7653 , respectively, and the partial estimates of $b$ ranged from 0.7556 to 0.7998 (Table 2). All of these are considerably less than 1.0. Whether this reflects inherent differences in the $I-S$ relationship between foliar and root diseases is not known, but further investigation of the $I-S$ relationship for other diseases caused by soilborne pathogens is merited.

When quantifying $I-S$ relationships, it is important to use a sufficiently large data set that represents the full range of $I$ and $S$ values that occur in actual disease epidemics. This is especially true if the predictive equation is to be used for disease assessment purposes. In our study, the $I-S$ relationship was derived from a set of seven independent data sets rather than a single large data set. The ranges of $I$ and $S$ values varied somewhat among the individual data sets, and no single data set contained the full range of possible $I$ and $S$ values. However, the combined data set does represent the full range of $I$ and $S$ values that are typically observed in epidemics of black root rot of pansy. The jackknife and cross-validation techniques proposed by Jones and Carberry (15) proved useful for estimating parameters and testing the performance of the model to describe the quantitative relationship between black root rot $I$ and $S$ across these independent data sets. Based on the jackknife-estimated 
and cross-validated parameters, $I$ appeared to be a reasonably accurate predictor of $S$.

In this study of black root rot of pansy, we have shown that the relationship between $I$ and $S$ for this root disease is similar to that of many foliar diseases. Based on the results of this study, we propose that $I$ assessed on discrete root segments is a more precise measure of black root rot intensity on pansy than assessments of $S$ on the entire root system. Equation 2, which is analogous to the Kono-Sugino equation, provided a good description of the $I-S$ relationship for black root rot of pansy. The prediction equations we derived should provide useful tools for disease assessment and serve as the basis for further investigation of $I-S$ relationships for diseases caused by other soilborne pathogens.

\section{LITERATURE CITED}

1. Adams, P. B. 1971. Effect of soil temperature and soil amendments on Thielaviopsis root rot of sesame. Phytopathology 61:93-97.

2. Anonymous. 1995. Diseases caused by Thielaviopsis basicola. Pages 30-32 in: Compendium of Flowering Potted Plant Diseases. M. L. Daughtrey, R. L. Wick, and J. L. Peterson, eds. American Phytopathological Society, St. Paul, MN.

3. Basu, P. K. 1988. Relationship between Phytophthora root rot severity index and the percentage of resistant alfalfa plants. Can. Plant Dis. Surv. 68:23-26.

4. Bateman, D. F., and Dimock, A. W. 1959. The influence of temperature on root rots of poinsettia caused by Thielaviopsis basicola, Rhizoctonia solani, and Pythium ultimum. Phytopathology 49:641-647.

5. Campbell, C. L., and Madden, L. V. 1990. Introduction to Plant Disease Epidemiology. John Wiley \& Sons, New York.

6. Chuang, T. Y., and Jeger, M. J. 1987. Relationship between incidence and severity of banana leaf spot in Taiwan. Phytopathology 77:15371541.

7. Copes, W. E., and Hendrix, F. F. 1996. Influence of $\mathrm{NO}_{3} / \mathrm{NH}_{4}$ ratio, $\mathrm{N}, \mathrm{K}$, and $\mathrm{pH}$ on root rot of Viola $\times$ wittrockiana caused by Thielaviopsis basicola. Plant Dis. 80:879-884.

8. Daamen, R. A. 1986. Measures of disease intensity in powdery mildew (Erysiphe graminis) of winter wheat. 2. Relationships and errors of estimation of pustule number, in- cidence and severity. Neth. J. Plant Pathol. 92:207-222.

9. Dillard, H. R., and Seem, R. C. 1990. Incidence-severity relationships for common maize rust on sweet corn. Phytopathology 80:842-846.

10. Giovannetti, M., and Mosse, B. 1980. An evaluation of techniques for measuring vesicular arbuscular mycorrhizal infection in roots. New Phytol. 84:489-509.

11. Godfrey, G. H. 1934. Indicator plants for measuring soil populations of the root-knot nematode, Heterodera marioni (Cornu) Goodey. Soil Sci. 38:3-27.

12. Holtz, B. A., and Weinhold, A. R. 1994. Thielaviopsis basicola in San Joaquin Valley soils and the relationship between inoculum density and disease severity of cotton seedlings. Plant Dis. 78:986-990.

13. James, W. C., and Shih, C. S. 1973. Relationship between incidence and severity of powdery mildew and leaf rust on winter wheat. Phytopathology 63:183-187.

14. Johnson, J. 1916. Host plants of Thielavia basicola. J. Agric. Res. 6:289-300.

15. Jones, P. N., and Carberry, P. S. 1994. A technique to develop and validate simulation models. Agric. Syst. 46:427-442.

16. Keller, J. R., and Potter, H. S. 1954. Thielaviopsis associated with root rot of some ornamental plants. Plant Dis. Rep. 38:354-358.

17. Kono, T., and Sugino, T. 1958. On the estimation of the density of rice stems infested by the rice stem borer. Japanese J. Appl. Entomol. Zool. 2:184-188.

18. Kranz, J. 1988. Measuring plant disease. Pages 35-50 in: Experimental Techniques in Plant Disease Epidemiology. J. Kranz and J. Rotem, eds. Springer-Verlag, Berlin.

19. Lindow, S. E. 1983. Estimating disease severity of single plants. Phytopathology 73:15761580.

20. Mayee, C. D., and Datar, V. V. 1989. Measuring plant disease. Pages 485-496 in: Perspectives in Plant Pathology. V. P. Agrihotri, N. Singh, H. S. Chaube, U. S. Singh, and T. S. Dwivedi, eds. Today and Tomorrow's Printers \& Publishers, New Delhi, India.

21. McRoberts, N., Hughes, G., and Madden, L. V. 2003. The theoretical basis and practical application of relationships between different disease intensity measurements in plants. Ann. Appl. Biol. 142:191-211.

22. Nachman, G. 1981. A mathematical model of the functional relationship between density and spatial distribution of a population. J. Animal Ecol. 50:453-460.

23. Nutter, F. W., Jr., Gleason, M. L., Jenco, J. H., and Christians, N. C. 1993. Assessing the ac- curacy, intra-rater repeatability, and inter-rater reliability of disease assessment systems. Phytopathology 83:806-812.

24. O'Brien, R. D., and van Bruggen, A. H. C. 1992. Accuracy, precision, and correlation to yield loss of disease severity scales for corky root of lettuce. Phytopathology 82:91-96.

25. Ogawa, K., Trigoe, T., and Amano, T. 1992. Relationship between disease incidence and disease severity in the assessment of club root on cabbage. Proc. Kanto Tosan Plant Prot. Soc. 39:103-106.

26. Pataky, J. K., and Headrick, J. M. 1988. Relationships between common rust incidence and severity on a susceptible and a partially resistant sweet corn hybrid. Phytopathology 78:1155-1160.

27. Rouse, D. I., Mackenzie, D. R., Nelson, R. R. and Elliott, V. J. 1981. Distribution of wheat powdery mildew incidence in field plots and relationship to disease severity. Phytopathology 71:1015-1020.

28. Seem, R. C. 1984. Disease incidence and severity relationships. Annu. Rev. Phytopathol. 22:133-150.

29. Seem, R. C., and Gilpatrick, J. D. 1980. Incidence and severity relationships of secondary infections of powdery mildew on apple. Phytopathology 70:851-854.

30. Suparyono, and Pataky, J. K. 1989. Relationships between incidence and severity of Stewart's and Goss's bacterial wilts and yield of sweet corn hybrids. Crop Prot. 8:363-368.

31. Tinline, R. D., Diehl, J. A., and Spurr, D. T. 1994. Assessment of methods for evaluating common root rot in spring wheat and infection of subterranean plant parts by the causal fungus Cochliobolus sativus. Can. J. Plant Pathol. 16:207-214.

32. Van den Berg, C. G. J., Rimmer, S. R., and Parks, P. 1993. Comparison of scales for rating severity of blackleg in canola. Can. J. Plant. Pathol. Rev. 15:49-53

33. Waggoner, P. E., and Rich, S. 1981. Lesion distribution, multiple infection, and the logistic increase of plant disease. Proc. Natl. Acad. Sci. USA 78:3292-3295.

34. Wilkinson, C. A., Shew, H. D., and Rufty, R. C. 1995. Evaluation of components of partial resistance to black root rot in burley tobacco. Plant Dis. 79:738-741.

35. Willocquet, L., and Savary, S. 2004. An epidemiological simulation model with three scales of spatial hierarchy. Phytopathology 94:883-891.

36. Xu, X. M., and Madden, L. V. 2002. Incidence and density relationships of powdery mildew on apple. Phytopathology 92:1005-1014 